\title{
Phytoprotection
}

\section{Une note sur la résistance à l'ergot chez le blé tendre, le blé dur et le triticale}

\section{Pageau, J. Collin et J.-M. Wauthy}

Volume 75, numéro 1, 1994

URI : https://id.erudit.org/iderudit/706051ar

DOI : https://doi.org/10.7202/706051ar

Aller au sommaire du numéro

Éditeur(s)

Société de protection des plantes du Québec (SPPQ)l

ISSN

0031-9511 (imprimé)

1710-1603 (numérique)

Découvrir la revue

Citer cet article

Pageau, D., Collin, J. \& Wauthy, J.-M. (1994). Une note sur la résistance à l'ergot chez le blé tendre, le blé dur et le triticale. Phytoprotection, 75(1), 45-49.

https://doi.org/10.7202/706051ar
Résumé de l'article

Un essai d'une durée de trois ans a permis d'évaluer la sensibilité de cultivars de céréales au champignon responsable de l'ergot (Claviceps purpurea). Les cultivars ont été évalués sous les conditions naturelles d'infection caractéristiques de la région du Lac Saint-Jean, au Québec. Lestriticales (X Triticosecale) ont montré une plus grande sensibilité à l'ergot que les blés durs (Triticum durum), qui à leur tour ont été plus sensibles que les blés tendres (Triticum aestivum). Le triticale cv. Bura était plus résistant à l'ergot que le cultivar Beaguelita. Le cultivar de blé dur Medora était plus résistant que le cultivar Kyle. Aucune différence significative n'a été observée parmi huit cultivars de blé tendre. La résistance à l'ergot est donc un facteur important à considérer dans le choix d'une espèce ou d'un cultivar destiné à une région où l'ergot est endémique. 


\title{
Une note sur la résistance à l'ergot chez le blé tendre, le blé dur et le triticale
}

\author{
Denis Pageau', Jean Collin² et Jean-Marie Wauthy ${ }^{1}$
}

Reçu 1993-04-07; accepté 1993-10-20

Un essai d'une durée de trois ans a permis d'évaluer la sensibilité de cultivars de céréales au champignon responsable de l'ergot (Claviceps purpurea). Les cultivars ont été évalués sous les conditions naturelles d'infection caractéristiques de la région du Lac Saint-Jean, au Québec. Les triticales (X Triticosecale) ont montré une plus grande sensibilité à l'ergot que les blés durs (Triticum durum), qui à leur tour ont été plus sensibles que les blés tendres (Triticum aestivum). Le triticale cv. Bura était plus résistant à l'ergot que le cultivar Beaguelita. Le cultivar de blé dur Medora était plus résistant que le cultivar Kyle. Aucune différence significative n'a été observée parmi huit cultivars de blé tendre. La résistance à l'ergot est donc un facteur important à considérer dans le choix d'une espèce ou d'un cultivar destiné à une région où l'ergot est endémique.

Pageau, D., J. Collin, and J.-M. Wauthy. 1994. A note on the resistance of soft wheat, durum wheat and triticale to ergot. PHYTOPROTECTION 75: 45-49.

A three-year study was conducted to evaluate the susceptibility of cereal cultivars to the fungus Claviceps purpurea. The cultivars were evaluated under the natural conditions of infection found in the Lac Saint-Jean region in Québec. The triticale ( $X$ Triticosecale) cultivars were more susceptible to ergot than the durum wheat (Triticum durum) cultivars, which were in turn more susceptible than the soft wheat (Triticum aestivum) cultivars. The triticale cv. Bura was more resistant to ergot than cultivar Beaguelita. The durum wheat cultivar Medora was more resistant than cultivar Kyle. No significant difference was observed among the soft wheat cultivars. Ergot resistance is thus an important factor to consider in the choice of a cultivar or a species to be grown in a region where ergot is endemic.

L'ergot, une maladie provoquée par le champignon Claviceps purpurea(Fr.) Tul., peut affecter toutes les graminées (Lorenz 1979; Mantle et al. 1977). La résistance à l'ergot a pu être observée chez des cultivars de blé tendre (Triticum aestivum L.) (Bauer 1972; Bretag et Merriman 1980; Darlington et Mathre 1976; Platford et Bernier 1976), de blé dur (Triticum

1. Agriculture Canada, Ferme expérimentale, Normandin (Québec), Canada GOW 2E0

2. Université Laval, Département de phytologie, Québec (Québec), Canada G1K 7P4 durum Desf.) (Platford et Bernier 1976), d'orge (Hordeum vulgare L.) (Cunfer et al. 1975) et de seigle de printemps (Secale cereale L.) (Sosulski et Bernier 1975).

La plupart de ces études ont été effectuées sous inoculation artificielle. Cependant, les travaux de Bauer (1972) effectués sous inoculation naturelle, ont démontré que certains cultivars de blé sont plus tolérants que d'autres à l'ergot. Au Québec, les cultivars sont recommandés en fonction de leurs qualités agronomiques désirables et leur résistance à certaines maladies. Cependant, aucune donnée ne permet 
d'identifier la réaction à l'ergot de ces différents cultivars sous des conditions naturelles d'infection.

Dans la région du Saguenay-Lac-SaintJean (Québec), c'est l'orge qui est l'espèce la plus touchée par l'ergot avec la destruction d'environ 2000 ha au cours de la période 1987-1991. Cependant, pour la même période, près de 100 ha de blé ont également été détruits. En 1987 et 1991, deux années où les niveaux d'infestation étaient très élevés dans la région, c'est près de $10 \%$ des superficies en blé qui ont été affectées par la maladie. Cette étude a donc été entreprise afin d'évaluer la résistance à l'ergot de différents cultivars de blé et de triticale ( $X$ TriticosecaleWittmack) recommandés ou candidats à la recommandation pour culture au Québec, en vue de déterminer la valeur des sources de résistance dans une région où l'ergot est une maladie importante.

Cet essai a été réalisé de 1988 à 1990 à quatre sites localisés au nord-ouest du Lac-Saint-Jean (Québec). Les quatre sites choisis pour l'étude étaient Saint-Eugène, Saint-Edmond, Normandin et la Ferme expérimentale d'Agriculture Canada à Normandin. À chaque année, les cultivars de blé et de triticale, faisant partie des essais de recommandation du Conseil des productions végétales du Québec, ont été évalués afin d'établir leur résistance à l'ergot. En 1988, huit cultivars de blé tendre (Casavant, Columbus, Katepwa, Laval-19, Messier, Milton, Mondor et Opal), deux cultivars de blé dur (Kyle et Medora) et un cultivar de triticale (Beaguelita) ont été évalués. En 1989 et 1990, le triticale cv. Bura s'est ajouté à la liste des cultivars évalués.

Les parcelles étaient constituées de quatre rangs d'une longueur de $6 \mathrm{~m}$, sauf à la Ferme expérimentale de Normandin où la longueur des parcelles était de 3,5 $\mathrm{m}$. L'écartement entre les rangs était de $18 \mathrm{~cm}$. Avant le semis, une application de $80 \mathrm{~kg} \mathrm{ha}^{-1} \mathrm{~d}^{\prime}$ azote (nitrate d'ammonium) et $30 \mathrm{~kg} \mathrm{ha}^{-1}$ de $\mathrm{P}_{2} \mathrm{O}_{5}$ (superphosphate) et de $\mathrm{K}_{2} \mathrm{O}$ (muriate de potassium) a été effectuée. La dose de semis a été de 340 grains viables $\mathrm{m}^{-2}$. Au stade trois feuilles des céréales, on a appliqué, à raison de $1,0 \mathrm{~L} \mathrm{ha}^{-1}$ un mélange composé de $280 \mathrm{~g}$ m.a. $L^{-1}$ de 2,4-D [acide (2,4-dichloro- phénoxy) acétique], de $80 \mathrm{~g} \mathrm{~m} \cdot \mathrm{a} \cdot \mathrm{L}^{-1}$ de mécoprop [acide (2-(4-chloro-2-méthylphénoxy)) propionique] et de $110 \mathrm{~g} \mathrm{~m}$.a. $\mathrm{L}^{-1}$ de dicamba [acide 3,6-dichloro-2méthoxybenzoique] pour lutter contre les mauvaises herbes dicotylédones. Les graminées adventices étaient absentes des sites d'essai.

Les deux rangs du centre de chaque parcelle ont été récoltés sur une longueur de $5,5 \mathrm{~m}$, sauf à la Ferme expérimentale de Normandin où les quatre rangs de chacune des parcelles ont été récoltés sur une longueur de $3 \mathrm{~m}$. Aucune inoculation de la maladie n'a été effectuée, les plantes étant soumises à l'infection naturelle caractéristique de la région.

La détermination du contenu en ergot a été effectuée à partir de deux échantillons d'environ $200 \mathrm{~g}$ de grain provenant de la récolte des parcelles. Les sclérotes et les grains ont été séparés à la main et pesés. Le contenu en ergot a été exprimé en pourcentage de poids: [poids des sclérotes/(poids des sclérotes + poids du grain) $] \times 100$.

Le dispositif expérimental utilisé à chaque site était constitué de trois blocs complets aléatoires. L'analyse statistique a été effectuée sur les données transformées $[\log (x+1)]$ et le test de comparaisons multiples de Tukey a été utilisé pour comparer les moyennes. Les coefficients de corrélation de Spearman ont été calculés entre le contenu en ergot et divers paramètres agronorniques (épiaison, hauteur des plants et rendement en grain) pour l'ensemble des espèces et des cultivars.

Les contenus en sclérotes observés chez les cultivars de triticale et de blé sont présentés au tableau 1. Fuisque I'objectif de l'essai était de mettre en valeur les différences génétiques entre les cultivars, et que ceux-ci expliquent la plus grande partie de la variation du contenu en ergot $\left(R^{2}=0,41\right)$, nous ne présentons que les moyennes observées pour les cultivars sur l'ensemble des combinaisons sites-années. Les interactions observées entre les années et les sites sont surtout causées par des variations du niveau d'infection naturel d'ergot. Plus l'infection est importante, plus les différences observées entre le 
triticale, les blés durs et les blés tendres sont marquées, alors que les blés tendres se comportent tous de façon assez similaire.

D'après Martens et al. (1984), une concentration de sclérotes dépassant $0,1 \%$ peut être considérée comme dangereuse pour l'alimentation du bétail. Le triticale cv. Beaguelita s'est donc avéré très sensible à l'ergot avec un contenu moyen en sclérotes de $2,68 \%$. Le cultivar de triticale Bura, qui a été introduit les deux dernières années de l'essai, s'est montré significativement plus tolérant que le cultivar Beaguelita. Le contenu moyen en sclérotes pour les deux dernières années était de $3,60 \%$ pour le cultivar Beaguelita comparativement à $2,01 \%$ pour le cultivar Bura. Platford et Bernier (1976), qui ont évalué la sensibilité à l'ergot du triticale, n'ont observé aucune différence du niveau de résistance entre six cultivars de triticale. Toutefois, Gregory et al. (1985) ont observé que certains cultivars de triticale étaient plus résistants que d'autres à l'ergot.

Parmi les deux cultivars de blé dur, le cultivar Kyle s'est montré beaucoup plus sensible à l'ergot que le cultivar Medora. Le contenu moyen en sclérotes observé

Tableau 1. Contenu moyen en sclérotes observé chez huit cultivars de blé tendre, deux cultivars de blé dur et un cultivar de triticale (1988-1990)

\begin{tabular}{|c|c|c|}
\hline Cultivar & $\begin{array}{c}\text { Contenu en sclérotes } \\
(\%)\end{array}$ & $\begin{array}{c}\text { Moyenne } \\
(\%)\end{array}$ \\
\hline Triticale & & 2,68 \\
\hline Beaguelita & $2,68 a^{a}$ & \\
\hline Blé dur & & 0,60 \\
\hline Kyle & $0,97 \mathrm{~b}$ & \\
\hline Medora & $0,23 \mathrm{c}$ & \\
\hline Blé tendre & & 0,10 \\
\hline Columbus & $0,16 \mathrm{~cd}$ & \\
\hline Mondor & $0,15 \mathrm{~cd}$ & \\
\hline Laval-19 & $0,13 \mathrm{~cd}$ & \\
\hline Milton & $0,09 \mathrm{~d}$ & \\
\hline Casavant & $0,08 d$ & \\
\hline Messier & $0,07 \mathrm{~d}$ & \\
\hline Katepwa & $0,07 \mathrm{~d}$ & \\
\hline Opal & $0,06 \mathrm{~d}$ & \\
\hline Tous les cultivars & & 0,43 \\
\hline \multicolumn{3}{|l|}{ Analyse de variance } \\
\hline Source & Valeur F & \\
\hline Cultivar & $242,3^{* *}$ & \\
\hline Année $X$ cultivar & $30,9^{* *}$ & \\
\hline Site $X$ cultivar & $17,9^{* *}$ & \\
\hline Année $X$ site $X$ cultivar & $9,6^{* *}$ & \\
\hline
\end{tabular}

** Significatif à $P \leq 0,01$.

a L'analyse a été effectuée après transformation logarithmique des données $[\log (x+1)]$. Les moyennes non transformées sont présentées. Les moyennes suivies d'une même lettre ne sont pas significativement différentes à $P \leq 0,05$ selon le test de Tukey. 
chez les cultivars de blé tendre a varié de 0,06 à $0,16 \%$. Cependant, les différences observées n'étaient pas statistiquement significatives. Néanmoins, Opal a été le cultivar qui a obtenu le plus faible contenu en sclérotes avec $0,06 \%$, tandis que le contenu en sclérotes du cultivar Columbus atteignait $0,16 \%$.

Les résultats démontrent que le triticale est plus sensible à l'ergot que les blés durs et les blés tendres. Le taux de pollinisation croisée plus élevé du triticale le rend probablement plus sensible à I'ergot que le blé tendre. Les travaux de Coley-Smith et Watkinson (1987) effectués avec inoculation artificielle avaient démontré que le triticale était plus sensible à l'ergot que le blé tendre. Cependant, ils mentionnent que le triticale est moins sensible à l'ergot que les blés durs. Nos résultats, au contraire, démontrent que les triticales étudiés sous inoculation naturelle sont plus sensibles à l'ergot que les blés durs de l'essai.

L'introduction de nouveaux cultivars de triticale au cours des prochaines années pourrait permettre de mieux évaluer la sensibilité à l'ergot des lignées de triticale candidates à la recommandation pour culture au Québec. Gregory et al. (1985) mentionnent qu'un programme de sélection du triticale favorisant des rendements élevés et un faible indice de tallage serait bénéfique pour la résistance à l'ergot car les épillets produits sur les talles ont tendance à être plus souvent stériles, ce qui accroît les risques de production de sclérotes.

Les deux cultivars de blé dur se sont montrés significativement plus tolérants à l'ergot que le triticale, mais plus sensibles que l'ensemble des blés tendres. Rapilly (1968) ainsi que ColeySmith et Watkinson (1987) mentionnent que le blé dur est plus sensible à l'ergot que le blé tendre.

Les cultivars de blé tendre évalués ont tous montré des niveaux de résistance à l'ergot comparables entre eux mais supérieurs à ceux des cultivars de blé dur et de triticale. Cependant, Platford et Bernier (1976) avaient observé des différences de tolérance à l'ergot entre des cultivars de blé tendre.
Les coefficients de corrélation avec le contenu en ergot étaient de $-0,4.3(P \leq$ $0,0001)$ pour la date d'épiaison, $-0,37$ $(P \leq 0,0001)$ pour la hauteur et de $-0,24$ $(P \leq 0,0001)$ pour le rendement en grain. Dans tous les cas, les coefficients de corrélation étaient significatifs mais faibles. Les coefficients de corrélation montrent qu'une faible augmentation de l'infection par l'ergot était associée à une épiaison hâtive ou à une taille plus courte. On a aussi observé une faible corrélation négative entre le contenu en ergot et le rendement en grain, le rendement des plantes infectées ayant tendance à être légèrement moins élevé que celui des plantes saines. Les travaux de Gregory et al. (1985) chez le triticale démontrent également une faible corrélation entre le contenu en sclérotes de la récolte et le rendement, la hauteur et la date de I'anthèse.

Des travaux similaires ont également démontré la présence de variabilités génétiques pour la résistance à l'ergot chez l'orge (Pageau et al. 1994). La résistance à l'ergot est donc un facteur important à considérer dans le choix d'une espèce ou d'un cultivar destiné à une région où l'ergot est endémique. L'utilisation de cultivars résistants permet de réduire le contenu en sclérotes lorsque les risques d'infection par l'ergot sont élevés. Ainsi, la culture du blé tendre devrait être favorisée au détriment du triticale et du blé dur dans les zones où la présence d'ergot est fréquente.

\section{REMERCIEMENTS}

Cette étude a été rendue possible grâce à une subvention de recherche provenant de I'Entente auxiliaire Canada-Québec sur le développement agro-alimentaire. Les auteurs remercient également Maurice Roy et Normand Dallaire pour leur aide technique.

\section{RÉFÉRENCES}

Bauer, A. 1972. The incidence of ergot in spring wheat varieties supplied with several fertilizer nitrogen rates. North Dakota Res. Rep. 39: 3-12. 
Bretag, T.W. et P.R. Merriman. 1980. Evaluation of Victorian wheat cultivars for resistance to ergot (Claviceps purpurea). Australas. Plant Pathol. 9: 111-112.

Coley-Smith, J.R. et L. Watkinson. 1987. Susceptibility of durum wheat cultivars to ergot disease. Ann. Appl. Biol. 110 (suppl.): 162-163.

Cunfer, B., D.E. Mathre et E.A. Hockett. 1975. Factors influencing the susceptibility of male-sterile barley to ergot. Crop Sci. 15: 194-196.

Darlington, L.C. et D.E. Mathre. 1976. Resistance of male sterile wheat to ergot as related to pollination and host genotype. Crop Sci. 16: 728-730.

Gregory, R.S., P.J. Webb et P.R. Hampson. 1985. Selection for resistance to ergot in triticale. Genetics and breeding of triticale, EUCARPIA meeting, Clermont-Ferrand, 25 July 1984, INRA, Paris. p. 551-558.

Lorenz, K. 1979. Ergot on cereal grains. Crit. Rev. Food Sci. Nutr. 11: 311-354.
Mantle, P.G., S. Shaw et D.A. Doling. 1977. Role of weed grasses in the etiology of ergot disease in wheat. Ann. Appl. Biol. 86: 339-351.

Martens, J.W., W.L. Seaman et T.G. Atkinson. 1984. Diseases of field crops in Canada. Can. Phytopathol. Soc., Harrow, ON. 160 pp.

Pageau, D., J. Collin et J.M. Wauthy. 1994. Evaluation of barley cultivars for resistance to ergot fungus, Claviceps purpurea (Fr.) Tul. Can. J. Plant Sci. (sous presse).

Platford, R.G. et C.C. Bernier. 1976. Reaction of cultivated cereals to Claviceps purpurea. Can. J. Plant Sci. 56: 51-58.

Rapilly, F. 1968. Études sur l'ergot du blé: Claviceps purpurea (Fr.) Tul. Ann. Épiphyt. 19: 305-329.

Sosulski, F. et C.C. Bernier. 1975. Ergot tolerance in spring rye. Can. Plant Dis. Surv. 55: 155-157. 